\title{
Voltage-time dilemma of pure electronic mechanisms in resistive switching memory cells
}

Herbert Schroeder, Victor V. Zhirnov, Ralph K. Cavin, and Rainer Waser

Citation: Journal of Applied Physics 107, 054517 (2010);

View online: https://doi.org/10.1063/1.3319591

View Table of Contents: http://aip.scitation.org/toc/jap/107/5

Published by the American Institute of Physics

\section{Articles you may be interested in}

Electrode kinetics of $\mathrm{Cu}-\mathrm{SiO}_{2}$-based resistive switching cells: Overcoming the voltage-time dilemma of electrochemical metallization memories

Applied Physics Letters 94, 072109 (2009); 10.1063/1.3077310

Resistive switching phenomena: A review of statistical physics approaches

Applied Physics Reviews 2, 031303 (2015); 10.1063/1.4929512

Resistive switching mechanism of $\mathrm{TiO}_{2}$ thin films grown by atomic-layer deposition

Journal of Applied Physics 98, 033715 (2005); 10.1063/1.2001146

Solid-state thin-film memistor for electronic neural networks

Journal of Applied Physics 67, 3132 (1998); 10.1063/1.345390

Characteristics and transport mechanisms of triple switching regimes of $\mathrm{TaO}_{\mathrm{x}}$ memristor Applied Physics Letters 110, 173504 (2017); 10.1063/1.4982593

Investigating the origins of high multilevel resistive switching in forming free $\mathrm{Ti} / \mathrm{TiO}_{2}-\mathrm{x}^{-}$based memory devices through experiments and simulations

Journal of Applied Physics 121, 094501 (2017); 10.1063/1.4977063

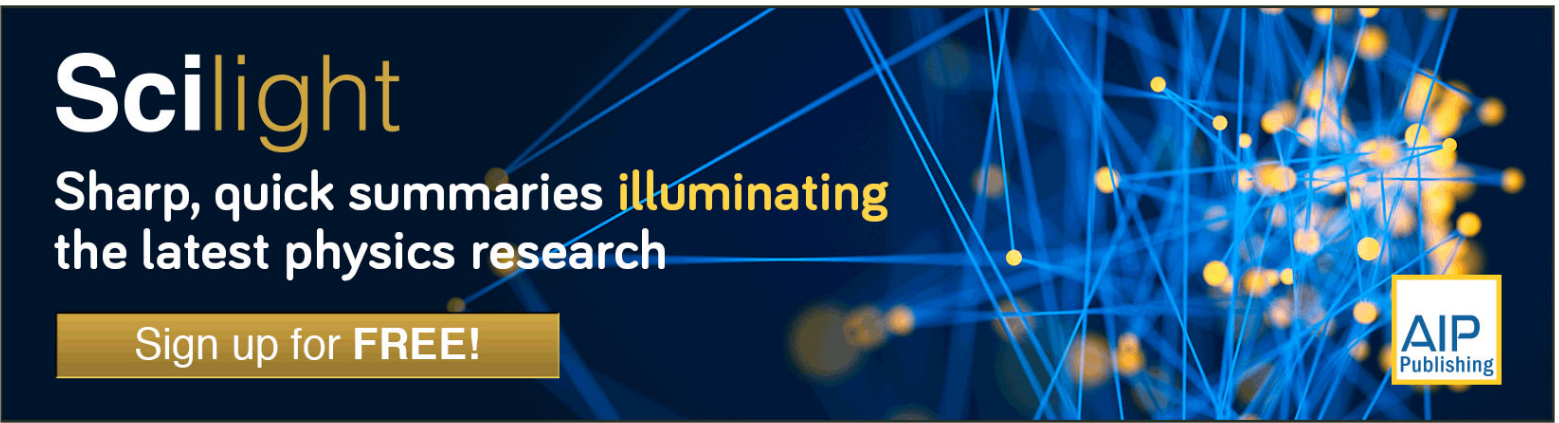




\title{
Voltage-time dilemma of pure electronic mechanisms in resistive switching memory cells
}

\author{
Herbert Schroeder, ${ }^{1, a)}$ Victor V. Zhirnov, ${ }^{2}$ Ralph K. Cavin, ${ }^{2}$ and Rainer Waser ${ }^{1}$ \\ ${ }^{1}$ IEM, Institut für Festkörperforschung and JARAFIT-Jülich Aachen Research Alliance Fundamentals of \\ Future Information Technology, Forschungszentrum Jülich GmbH, D-52425 Jülich, Germany \\ ${ }^{2}$ Semiconductor Research Corporation, Durham, North Carolina 27703, USA
}

(Received 19 October 2009; accepted 19 January 2010; published online 11 March 2010)

\begin{abstract}
Metal/insulator/metal thin film stacks showing stable resistive switching are promising candidates for future use as a nonvolatile resistive random access memory, competitive to FLASH and DRAM. Although the switching mechanisms are not completely understood a lot of theories and models try to describe the effects. One of them postulates the trapping and detrapping of electronic charge in immobile traps as the reason for the resistance changes, also known as Simmons \& Verderber model. This contribution shows that this "pure electronic" switching mechanism will face a voltage-time dilemma-general to all switching insulators-at conditions competitive to the state-of-the-art FLASH. There is an incompatibility between the long retention time (10 years) and the short READ/WRITE current pulses $\left(\mathrm{t}_{\mathrm{READ} / \mathrm{WRITE}} \leq 100 \mathrm{~ns}\right)$ at high densities (area $\leq 100$ $\left.\times 100 \mathrm{~nm}^{2}\right)$ at low applied voltages $(\leq 1 \mathrm{~V})$. This general dilemma is exemplified in two detailed scenarios with different electronic band and defect properties. (C) 2010 American Institute of Physics. [doi:10.1063/1.3319591]
\end{abstract}

\section{INTRODUCTION}

Thin film stacks of an insulator (e.g., an oxide) between metal electrodes [Metal/insulator/metal (MIM) stacks] often show unipolar and/or bipolar resistance switching, i.e., the system has at least two different, stable resistance states between which the system can be switched by application of an electrical field (voltage or current controlled). The change from the high resistance state (HRS, OFF-state) into the low resistance state (LRS, ON-state) is called SET and the opposite change is RESET. If both of these switchings can be done with either polarity of the electric field it is called unipolar resistive switching, if SET and RESET switchings need opposite polarity it is named bipolar resistive switching. Due to their simple geometry and the stability of the resistance states such stacks have a significant potential as future ultra large scale integrated, nonvolatile memories, called resistive random access memory (ReRAM), and may compete with, e.g., DRAM or FLASH.

Because of this promise, such MIM stacks have been extensively investigated for approximately forty years with a surge of studies in the last decade. To explain the switching mechanism some of the various suggested models are based on a homogeneous band description and capture and release of electronic charges in localized traps of the dielectric. Simmons and Verderber ${ }^{1}$ were one of the first to use this "electronic" model to explain their experimental switching results on large scale gold/silicon-monoxide/aluminum $(\mathrm{Au} / \mathrm{SiO} / \mathrm{Al})$ stacks, and their interpretation has been adopted for many other MIM material systems [e.g., Refs. 2-5].

It is demonstrated in this contribution that for conditions competitive with or better than "state-of-the-art" FLASH memories this pure "electronic" mechanism for resistive

${ }^{a)}$ Electronic mail: he.schroeder@fz-juelich.de. switching of a thin film ReRAM will result in a dilemma: The requirements for a very large retention time for ReRAM devices on one hand and for short READ/WRITE times with high current densities at low applied voltage on the other hand are shown to be contradictory. This general dilemma will be described for two scenarios of MIM stacks.

\section{THE PURE ELECTRONIC MODEL AND ITS BENCHMARK DATA}

While in a DRAM the charge itself, stored in a capacitor, is used for the memory effect, in FLASH and in the proposed electronic ReRAM the stored charge is used to control the current through the device and thus its resistance. In a FLASH transistor the charge is stored in and released from a floating gate electrode thus controlling the induced charges in the transistor channel and hence its resistance, as sketched in Figs. 1(a). The current path for the READ operation through the transistor channel is different from that for the WRITE/ERASE operations which change the trapped charge (TC) in the floating gate electrode. Improvements of the re-

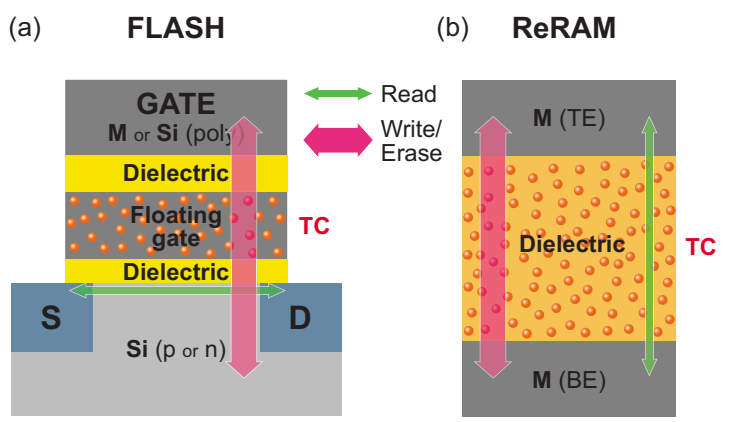

FIG. 1. (Color online) Comparison of TC based memories: (a) FLASH (1 Tr) and (b) ReRAM (gate capacitor only). Differences in current flow for READ and WRITE/ERASE are indicated by arrows. 
TABLE I. Benchmarks for a nonvolatile ReRAM competitive to FLASH. Numbers are for a temperature of 358 $\mathrm{K}\left(85^{\circ} \mathrm{C}\right)$.

\begin{tabular}{lcc}
\hline \hline Feature & Range & Driver \\
\hline Cell size, A & $<100 \times 100 \mathrm{~nm}^{2}=10^{-10} \mathrm{~cm}^{-2}$ & Cell scaling \\
Store time, $t_{\mathrm{S}}$ & $>3 \times 10^{8} \mathrm{~s}(=10 \mathrm{y})$ & Nonvolatility \\
Write time, $t_{\mathrm{W}}$ & $<10^{-7} \mathrm{~s}$ & \\
Read time, $t_{\mathrm{R}}$ & $<10^{-7} \mathrm{~s}$ & Competition with FLASH \\
Read voltage, $V_{\mathrm{R}}$ & $<1 \mathrm{~V}$ & \\
Read current, $I_{\mathrm{R}}$ & $\sim 10^{-6} \mathrm{~A}$ & Sensing of memory state \\
Read current density, $J_{\mathrm{R}}$ & $>10^{4} \mathrm{~A} / \mathrm{cm}^{2}$ & \\
Resistance ratio, $\mathrm{R}_{\mathrm{OFF}} / \mathrm{R}_{\mathrm{ON}}$ & $\sim 10$ & \\
\hline \hline
\end{tabular}

tention of this TC in a FLASH together with high current densities and hence low times for the WRITE/ERASE operations (as, e.g., by the "crested" barriers proposed by Likharev ${ }^{6}$ ) will not work in the ReRAM stack because it will not allow the high current densities needed for the READ operations at low voltages (see below and Table I). This is in contrast to the FLASH, as in the ReRAM stack [see Figs. 1(b)], which can be regarded as a leaky capacitor, the current path for all the memory operations is the same, i.e., through the stack. This important difference results in a much simpler geometry and therefore high integration capabilities, but is one of the main reasons for the dilemma of a pure "electronic" ReRAM.

In order to be competitive with a "state of the art" FLASH memory, some benchmark data for the ReRAM are postulated and collected in Table I together with the reasons for the selection. The cell size for future devices should be smaller than $100 \times 100 \mathrm{~nm}^{2}=10^{-10} \mathrm{~cm}^{2}$, which is (much) smaller than the stack areas for most of the reported experimental data for ReRAM. The retention time for any nonvolatile memory should be larger than 10 years $\left(\approx 3 \times 10^{8} \mathrm{~s}\right)$ at a temperature of $85{ }^{\circ} \mathrm{C}(358 \mathrm{~K})$. The times for WRITE/ ERASE and READ operations were selected to be smaller than $100 \mathrm{~ns}$ at a read voltage less than $1 \mathrm{~V}$, an improvement relative to present-day FLASH memories. WRITE/ERASE pulses should be at voltages $\leq 10 \cdot \mathrm{V}_{\mathrm{READ}}$ in order to be comparable or better than FLASH. To allow for inexpensive, but accurate sensing of the memory state, the read current for one state should be $\approx 1 \mu \mathrm{A}$ with an OFF/ON ratio of the resistances states of at least ten. This results in a very large

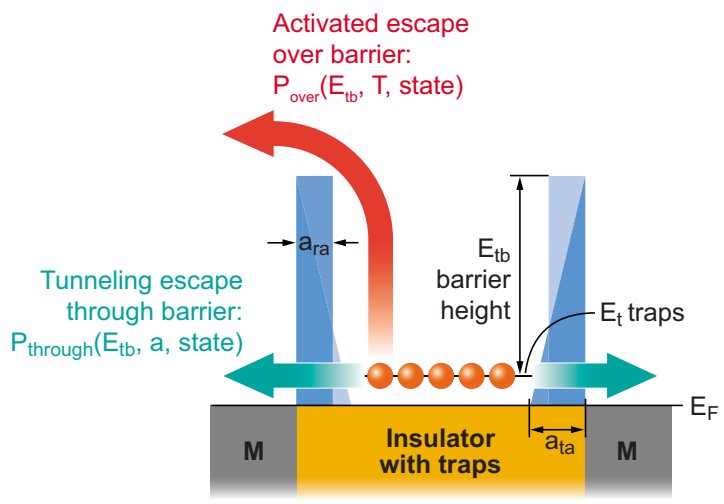

FIG. 2. (Color online) Scheme of trapped charge inside potential wells (rectangular, triangular) and possible escape paths. current density for READ of $>10^{4} \mathrm{~A} / \mathrm{cm}^{2}$ and an even larger current densities during the switching (i.e., during WRITE/ERASE pulses).

These benchmark data have far-reaching consequences for the electronic properties of the MIM stack with regard to the band structure of the (oxide) insulator (described as wide bandgap semiconductor), the kind of traps, and the selection of electrodes. This will be demonstrated with some examples.

The retention time of 10 year calls for potential wells around the trapped electronic charge of certain height and width. As sketched in Fig. 2 a trapped electronic charge has two paths for escape (except electron-hole recombination), one over the potential barrier and one through it via tunneling. Simple estimates show that for the activated jump over the barrier a height of about $1.5 \mathrm{eV}$ at $T=358 \mathrm{~K}\left(85^{\circ} \mathrm{C}\right)$ is necessary to keep the charge trapped with a probability of $p=0.5$ for the specified storage time. For the half-life times listed in Table II an attempt frequency $\mathrm{f}_{\text {attempt }}=10^{12} \mathrm{~Hz}$ (1-10 THz are typical frequencies for acoustic phonons) has been used with a Boltzmann activation term as shown in Table II.

The transmission probability for tunneling in the Wentzel, Kramers, and Brillouin (WKB) approximation, $T_{\mathrm{WKB}}$, for tunneling ${ }^{7}$ has been calculated for rectangular, trapezoidal, and triangular barriers of height(s) $\Phi_{B a}\left(\right.$ at $\left.x=a_{t}\right)$ and $\Phi_{B 0}$ (at $\left.x=0\right)$ (with one of them at least $1.5 \mathrm{~V}$ ) and a base width $a_{t}$. For an arbitrary energy profile $E(x)$ (measured in volts; the reference energy is assumed zero) this transmission coefficient is

TABLE II. Trap retention time $t_{\text {Ret }}$ (half life time: probability $p=0.5$ ) of thermally activated escape at $\mathrm{T}=85^{\circ} \mathrm{C}$ in dependence of effective barrier height $E_{b}^{\text {eff }}$.

\begin{tabular}{lcc}
\hline \hline $\begin{array}{c}\mathrm{E}_{\mathrm{b}}^{\text {eff }} \\
(\mathrm{eV})\end{array}$ & $\begin{array}{c}\mathrm{t}_{\text {Ret }} \\
(\mathrm{s})^{\mathrm{a}}\end{array}$ & $\begin{array}{c}\mathrm{t}_{\text {Ret }} \\
(\mathrm{y})\end{array}$ \\
\hline 0.25 & $1.66 \times 10^{-9}$ & $5.2 \times 10^{-17}$ \\
0.5 & $5.48 \times 10^{-6}$ & $1.7 \times 10^{-13}$ \\
1 & $6.01 \times 10^{+1}$ & $1.9 \times 10^{-6}$ \\
1.25 & $1.99 \times 10^{+5}$ & $6.3 \times 10^{-3}$ \\
1.5 & $6.60 \times 10^{+8}$ & 21
\end{tabular}

${ }^{\mathrm{a}}$ Calculated for $\mathrm{p}=\mathrm{t}_{\mathrm{Ret}}(\mathrm{dp} / \mathrm{dt})=0.5,(\mathrm{dp} / \mathrm{dt})=\mathrm{f}_{\text {attempt }} \exp \left(-\mathrm{E}_{\mathrm{tb}}^{\mathrm{eff}} / \mathrm{k}_{\mathrm{B}} \mathrm{T}\right)$ 


$$
\begin{aligned}
T_{\mathrm{WKB}} & =\exp \left[-\frac{2}{\hbar} \int_{0}^{a_{t}} d x \sqrt{2 m_{e}^{*}} \sqrt{e E(x)}\right] \\
& =\exp \left[-\frac{2 \sqrt{2 m_{e}^{*}} a_{t}}{\hbar} \frac{1}{a_{t}} \int_{0}^{a_{t}} d x \sqrt{e E(x)}\right] .
\end{aligned}
$$

For a rectangular shape $E(x)=$ const $=\Phi_{B a}=\Phi_{B 0}=\Phi_{B}$, the result is

$$
T_{\mathrm{WKB}}^{\mathrm{rect}}=\exp \left[-\frac{2 \sqrt{2 m_{e}^{*} e \Phi_{B}}}{\hbar} a_{t}\right]
$$

For a trapezoidal barrier with heights left $(x=0)$ and right $\left(x=a_{t}\right), \Phi_{B 0}$ and $\Phi_{B a}$, respectively, the transmission coefficient is

$$
T_{\mathrm{WKB}}^{\mathrm{trap}}=\exp \left[-\frac{2 \sqrt{2 m_{e}^{*}} a_{t} \frac{2}{\hbar}}{3} \sqrt{e} \frac{\Phi_{B a}^{3 / 2}-\Phi_{B 0}^{3 / 2}}{\Phi_{B a}-\Phi_{B 0}}\right] .
$$

The case of a triangle barrier (which is used as an approximation for the parabolic screening potential) is a special case of the trapezoidal barrier with one of the barriers, e.g., $\Phi_{B a}$ $=0$

$$
T_{\mathrm{WKB}}^{\mathrm{tri}}=\exp \left[-\frac{2 \sqrt{2 m_{e}^{*}} a_{t}}{\hbar} \frac{2}{3} \sqrt{e \Phi_{B 0}}\right] .
$$

For the tunneling escape the frequency of the quantummechanical ground state of a free electron $\left(m_{e}^{*}=m_{e 0}\right)$ in a potential well with a width of a lattice constant $(\approx 0.4 \mathrm{~nm})$, simulating a trap, has been chosen as attempt frequency, $\mathrm{f}_{\text {attempt }}=5 \times 10^{14} \mathrm{~Hz}$. For a tunneling probability of $\mathrm{p}_{\mathrm{t}} \leq 0.5$, a base width $a_{t} \geq 4$ and $a_{t} \geq 6 \mathrm{~nm}$ for the rectangular and triangular potential well, respectively, can be estimated to guarantee 10 year retention. In this estimation a maximum barrier heights of $e \Phi_{B}=1.5 \mathrm{eV}$ and $m_{e}^{*}=m_{e 0}$ were used. Therefore, trapped electronic charge has to be surrounded by potential wells of at least $1.5 \mathrm{eV}$ heights and 4 or $6 \mathrm{~nm}$ (base) thickness for rectangular or triangular barriers, respectively, to stay in the system for the requested time. If the effective electronic mass for thermionic emission or tunneling is different from that for free electrons the changed probabilities result in adjusted height and width of the potential well, but these effects will cancel out in the calculated tunneling current densities for READ and WRITE/ERASE (see Secs. III and IV).

It is worth noting that the assumed immobile traps for electronic charge (in our cases we assume electrons, but similar scenarios are valid for holes as well!) have certain characteristics, i.e., they are donorlike or acceptorlike. ${ }^{8}$ The traps introduced into the scenarios are postulated to be neutral if not filled and (negatively) charged when filled (with electrons). Such characteristic is acceptorlike. In general the equilibrium occupation of such traps follows the FermiDirac distribution function and is considered for the position of the equilibrium Fermi level of the system.

The conditions outlined in the previous sections can be realized in a MIM thin film stack in different ways. As examples, two extreme scenarios of symmetric MIM stacks

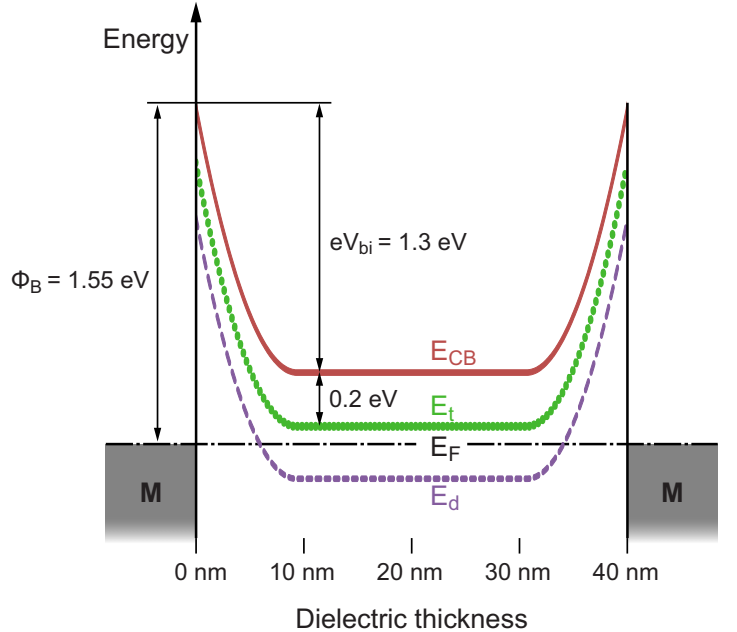

FIG. 3. (Color online) Possible band diagram with trapped charge in wide band gap materials: scenario no. 1, the "Simmons-like" case, i.e., (electronic) charge trapped in shallow and homogeneously distributed acceptorlike (electron) traps with appropriate interfacial potential wells (width and built-in voltage $\mathrm{V}_{\mathrm{bi}}$ due to donor doping) to prevent escape.

will be discussed in detail. Their equilibrium band diagrams (i.e., equilibration of the Fermi levels in the MIM stack) are sketched in Figs. 3 and 4, respectively, and their characteristic numbers are listed in Table III.

In the scenario no. 1 (see Fig. 3) the traps are rather shallow, $\mathrm{E}_{\mathrm{T}}=-0.2 \mathrm{eV}$ below the conduction band minimum $(\mathrm{CBM})$, i.e., these shallow traps are regarded as very deep
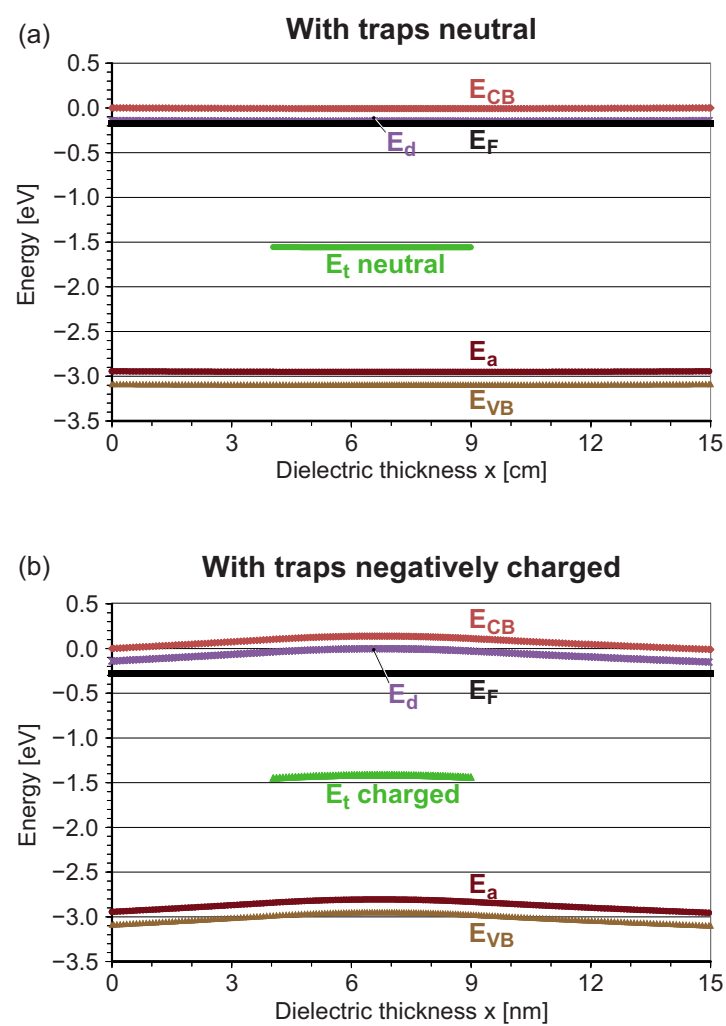

FIG. 4. (Color online) Possible band diagram with trapped charge in wide band gap materials: scenario no. 2, i.e., (electronic) charge trapped in deep and inhomogeneously distributed acceptorslike traps (for electrons), (a) traps neutral (nonequilibrium state), (b) traps (negatively) charged (equilibrium state). 
TABLE III. Characteristic numbers and properties for the materials in the thin film stacks (dielectrics with traps/defects, electrodes) for the two different scenarios (under equilibrium conditions).

\begin{tabular}{|c|c|c|}
\hline Property & Scenario no. 1 & Scenario no. 2 \\
\hline Dielectrics (used in examples) & $\mathrm{SiO} ; \mathrm{TiO}_{2} ; \mathrm{STO}$ & STO \\
\hline $\begin{array}{l}\text { Relative dielectric constants: } \mathrm{K} \text { (static), } \mathrm{K}_{\mathrm{opt}} \text { [at optical } \\
\text { frequencies }\left(=\mathrm{n}^{2}\right) \text { ] }\end{array}$ & $\begin{array}{c}\mathrm{SiO}: 4 ; 4 \\
\mathrm{TiO}_{2}: 40,7 \\
\text { STO: } 200,5.5\end{array}$ & $200,5.5$ \\
\hline Thickness d (nm) & 40 & 15 \\
\hline Temperature (K) & 358 & 358 \\
\hline Electron effective mass $m_{e}^{*}\left(=m_{e 0}\right.$ for all!) & $m_{e 0}$ & $m_{e 0}$ \\
\hline Band gap (eV) & $>3$ & 3.1 \\
\hline \multicolumn{3}{|l|}{$\begin{array}{l}\text { Defects (constant density throughout the dielectric } \\
\text { thickness if not stated otherwise): }\end{array}$} \\
\hline Donor defect density $\mathrm{N}_{\mathrm{D}}\left(\mathrm{cm}^{-3}\right)$ & $\begin{array}{c}\mathrm{SiO}: 1.6 \times 10^{19} \\
\mathrm{TiO}_{2}: 1.6 \times 10^{20} \\
\text { STO: } 8 \times 10^{20}\end{array}$ & $2 \times 10^{19}$ \\
\hline Donor defect energy $E_{D}$ below CBM $(e V)$ & -0.4 (for all) & -0.15 \\
\hline Acceptor defect density $\mathrm{N}_{\mathrm{A}}\left(\mathrm{cm}^{-3}\right)$ & 0 & $8 \times 10^{18}$ \\
\hline Acceptor defect energy $\mathrm{E}_{\mathrm{A}}$ below $\mathrm{CBM}(\mathrm{eV})$ & & -2.95 \\
\hline (Electron) trap density (acceptorlike) $\left(\mathrm{cm}^{-3}\right)$ & $2.5 \times 10^{18}$ & $\begin{array}{l}1.2 \times 10^{20} \\
(4-9 \mathrm{~nm})\end{array}$ \\
\hline Trap defect energy $\mathrm{E}_{\mathrm{T}}$ below $\mathrm{CBM}(\mathrm{eV})$ & -0.2 & -1.55 \\
\hline Equilibrium Fermi level $\mathrm{E}_{\mathrm{F}}$ below $\mathrm{CBM}(\mathrm{eV})$ & $\begin{array}{c}\text { SiO: }-0.27 \\
\mathrm{TiO}_{2}:-0.23 \\
\text { STO. }-0.21\end{array}$ & -0.18 \\
\hline Equilibrium trap state & Not charged & Charged \\
\hline \multicolumn{3}{|l|}{ Interface (Electrode) } \\
\hline Barrier height $e \Phi_{B}(\mathrm{eV})$ and type & 1.55; Schottky & 0.18 ; neutral \\
\hline Built-in voltage $e \mathrm{~V}_{\mathrm{bi}}(\mathrm{eV})$ & 1.3 & 0 \\
\hline Barrier height for traps $(\mathrm{eV})$ & 1.5 & 1.55 \\
\hline Barrier width for traps $(\mathrm{nm})$ at $\mathrm{V}_{\text {appl }}=0$ & 6 (triangular) & 4 (rectangular) \\
\hline
\end{tabular}

acceptors. Therefore, the necessary high potential well for the trapped charge has to be realized in the interface region at the electrodes with a large barrier, $e \Phi_{B}=1.55 \mathrm{eV}$ (which is - as a rule of thumb - the difference between the electrode work function, $\mathrm{E}_{\mathrm{f}}^{\mathrm{M}}$, and the electron affinity of the dielectric insulator, $\chi^{\mathrm{I}}$ ), and a large built-in voltage, $e \mathrm{~V}_{\mathrm{bi}}=1.3 \mathrm{eV}$, making the total barrier height for traps in the screened flat band region $1.5 \mathrm{eV}$, as requested. In order to create the built-in voltage at the interfaces, donor defects have to be introduced. For simplicity, in this example a single in-gap energy of the donor states was chosen at $E_{D}=-0.4 \mathrm{eV}$ below CBM. The donor density is determined by two limitations: it has to be large enough to give the requested screening $\left(\mathrm{V}_{\mathrm{bi}}\right)$ in (much) less than half of the dielectric insulator film thickness $(=40 \mathrm{~nm}$ for scenario no. 1), but it should be small enough to guarantee a screening thickness $\geq 6 \mathrm{~nm}$ of the "triangle"-like potential well at the interface (this approximates the parabolic potential shape due to the Poisson equation). For an insulator with a dielectric constant $\mathrm{K}=4$, a donor density of $\mathrm{N}_{\mathrm{D}}=1.6 \times 10^{19} \mathrm{~cm}^{-3}$ is sufficient as calculated by the Poisson equation with the abrupt defect density approximation. A trap density of $\mathrm{N}_{\mathrm{T}}=2.5 \times 10^{18} \mathrm{~cm}^{-3}$ at -0.2 $\mathrm{eV}$ together with the density and energy level of the donors will position the Fermi energy at $\mathrm{E}_{\mathrm{F}} \approx-0.25 \mathrm{eV}$ below CBM, as it is sketched in Fig. 3. Therefore, in equilibrium the majority of the acceptorlike traps and donors are un- charged in the flat band region due to Fermi-Dirac statistics. If the dielectric constant is different, the donor density has to be adjusted according to the ratio of the dielectric constants to result in the same screening length of $6 \mathrm{~nm}$, e.g., for $\mathrm{K}$ $=40\left(\mathrm{TiO}_{2}\right)$ and $\mathrm{K}=200\left(\mathrm{SrTiO}_{3}\right)$ the donor density is $\mathrm{N}_{\mathrm{D}}$ $=1.6 \times 10^{20}$ and $\mathrm{N}_{\mathrm{D}}=8 \times 10^{20} \mathrm{~cm}^{-3}$, respectively.

The band diagram for the scenario no. 2 is shown in Fig. 4. The dielectric insulator has a thickness of $15 \mathrm{~nm}$. For the case that the traps are not charged [Fig. 4(a)] it is characterized by flat bands and a small barrier at the interfaces of 0.17 $\mathrm{eV}$ between the electrode work function and the CBM due to the selected electrode and the partly self-compensating donor and acceptor defects as listed in Table III. It should be mentioned that the parameter set for these defects (densities and energies) is not unique for the same result. Due to this band diagram the possibly trapped charge has to be deep in the gap. For this scenario no. 2 the traps are assumed at $\mathrm{E}_{\mathrm{T}}=$ $-1.55 \mathrm{eV}$ below CBM (which is about mid-gap for materials such as $\mathrm{SrTiO}_{3}$ ), and they are inhomogeneously distributed-only between 4 and $9 \mathrm{~nm}$ apart from the left electrode-with a density of $\mathrm{N}_{\mathrm{T}}=1.2 \times 10^{20} \mathrm{~cm}^{-3}$, which corresponds to a charge of $10 \mu \mathrm{C} / \mathrm{cm}^{2}$. In contrast to scenario no. 1, the equilibrium state of these acceptorlike traps is the charged one $\left(\mathrm{E}_{\mathrm{T}}<\mathrm{E}_{\mathrm{F}}\right)$. This additional space charge changes the band diagram as shown in Fig. 4(b). 


\section{THE READ OPERATION}

According to the postulated benchmarks in Table I a READ current density, $\mathrm{I}_{\text {READ }} \geq 10^{4} \mathrm{~A} / \mathrm{cm}^{2}$, at an applied voltage, $\mathrm{V}_{\text {appl }} \leq 1 \mathrm{~V}$, is requested. This current density has to be delivered by the injecting electrode (cathode for electrons) by thermionic emission over the interface barrier into the conduction band of the dielectric insulator and/or by tunneling through the barrier into localized defect states and/or conduction band. After injection, the same current must be transported through the dielectric insulator (possibly in various conduction paths) and then ejected into the opposite electrode. This READ current should be different from transient currents such as charging the capacitance of the dielectric, so that such currents have to be much smaller at the maximum READ time, $\mathrm{t}_{\mathrm{READ}}<100$ ns.

The maximum geometrical capacitance of the 100 $\times 100 \mathrm{~nm}^{2}$ thin film stack is that of scenario no. 2 with thickness $d=15 \mathrm{~nm}$ and $\mathrm{K}=200$. Using the simple equation for a plate capacitor the capacitance is $\mathrm{C} \leq 1.17 \mathrm{fF}$ or a capacitance density of $\mathrm{C} / \mathrm{A}=11.7 \mu \mathrm{F} / \mathrm{cm}^{2}$. Using a line resistance of $\mathrm{R}_{\text {line }}=1 \mathrm{k} \Omega$ the time constant for charging is about $\tau_{\mathrm{RC}} \approx 10^{-12} \mathrm{~s}$. Assuming a Debye relaxation for the charging of the capacitor at READ conditions the current density can be expressed as

$$
\begin{aligned}
& \mathrm{j}_{\text {charging }}=\mathrm{j}_{0} \exp \left[\frac{-\mathrm{t}}{\tau_{\mathrm{RC}}}\right] \\
& \text { with } \mathrm{j}_{0}=\frac{\left(\frac{\mathrm{V}_{\text {appl }}}{\mathrm{R}_{\text {line }}}\right)}{\mathrm{A}_{\text {cell }}}=10^{7} \mathrm{~A} / \mathrm{cm}^{2} .
\end{aligned}
$$

For times $\mathrm{t}>3 \times 10^{-11} \mathrm{~s}\left(\ll \mathrm{t}_{\text {read }}\right)$ the current density $\mathrm{j}_{\text {charging }}$ is negligibly small due to the exponential decay.

In order to estimate the possible relaxation currents one can use experimental data at larger times for extrapolation into the time range for READ, $t_{\text {READ }} \leq 100 \mathrm{~ns}$. For STO the relaxation current densities are in the range $\leq 10^{-6} \mathrm{~A} / \mathrm{cm}^{2}$ at $1 \mathrm{s.}{ }^{9}$ Usually the relaxation currents versus time can be fitted by a power law with time exponents $\alpha$ between 0.5 and $1 .^{10}$ Using the upper limit $\alpha=1$ for the extrapolation to $10^{-8} \mathrm{~s}$ $\left(=0.1 \cdot t_{\text {READ }}\right)$ the upper bound for the relaxation current density is $\mathrm{j}_{\text {Relax }} \leq 10^{2} \mathrm{~A} / \mathrm{cm}^{2} \ll 10^{4} \mathrm{~A} / \mathrm{cm}^{2}=\mathrm{j}_{\text {READ }}$. This estimate has to be checked for every system, but the relaxation currents are usually smaller for low $\mathrm{K}$ materials.

The maximum current density over the barrier by thermionic emission at a temperature $\mathrm{T}=358 \mathrm{~K}$ can be estimated by the "Schottky" equation [i.e., the Richardson equation for emission from the electrode into the conduction band (CB) of the dielectric] including the barrier reduction $\Delta \Phi$ by the electric field at the injecting interface, the so-called Schottky-effect:

$$
\mathrm{j}_{\mathrm{Th} \mathrm{Em}}=\mathrm{A}^{\mathrm{eff}} T^{2} \exp \left[-\frac{e\left(\Phi_{B}-\Delta \Phi\right)}{k_{B} T}\right] .
$$

The barrier reduction is $\Delta \Phi=\left[e F(x \approx 0) / 4 \pi \varepsilon_{0} \varepsilon_{\mathrm{r}, \mathrm{opt}}\right]^{1 / 2}$ with $F$ the electrical field at the injecting interface $\left[\approx\left(V_{\mathrm{bi}}\right.\right.$ $\left.\left.+V_{\text {appl }} / 2\right) / a_{t}\right]$ and $\varepsilon_{\mathrm{r}, \text { opt }}$ the relative permittivity at optical frequencies, related to the refraction index $n=\left(\varepsilon_{\mathrm{r}, \mathrm{opt}}\right)^{1 / 2}$, $e$ the

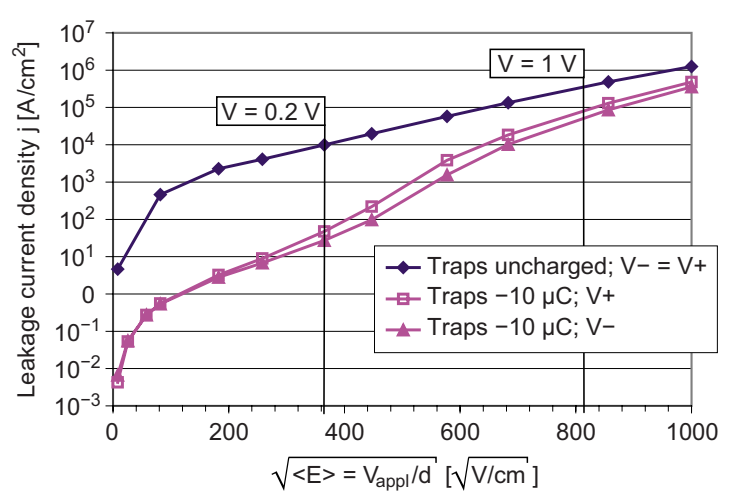

FIG. 5. (Color online) READ operation for scenario no. 2: steady state leakage current densities vs square root of mean applied field, $\langle\mathrm{E}\rangle^{1 / 2}$, ("Schottky-plot") for a M/STO (15 nm)/M thin film stack, i.e., charge trapped in deep and inhomogeneously distributed acceptorslike traps for electrons. Top: traps neutral; bottom: traps charged. Below $\mathrm{V}_{\text {appl }}<1 \mathrm{~V}$ the difference is a factor of $\geq 10$.

unit charge and $\varepsilon_{0}$ the vacuum permittivity. As this estimate should be applied to different dielectrics we have used the free electron mass instead of various effective masses in the Richardson constant, i.e., $\mathrm{A}^{\text {eff }}=\mathrm{A}=120 \mathrm{~A} \mathrm{~cm}^{-2} \mathrm{~K}^{-2}$, and the maximum $\mathrm{V}_{\text {appl }}=1 \mathrm{~V}$ for READ. Using an effective thickness of $12 \mathrm{~nm}$ (i.e., both screening regions in Fig. 3) for the voltage drop and, for example, an optical dielectric constant $\mathrm{K}_{\mathrm{opt}}(\mathrm{SiO})=4$ for the scenario no. 1 and a homogeneous field with $\mathrm{d}=15 \mathrm{~nm}$ [see Fig. 4(a)] and, for example, an optical dielectric constant $\mathrm{K}_{\mathrm{opt}}(\mathrm{STO})=5.5$ for the scenario no. 2 , the effective (reduced) barrier heights are 1.24 (no. 1) and 0.04 $\mathrm{eV}$ (no. 2), respectively, for the cases of uncharged traps. Application of Eq. (4) results in the corresponding maximum current densities of $6 \times 10^{-11}$ and $4 \times 10^{6} \mathrm{~A} / \mathrm{cm}^{2}$, respectively. As for scenario no. 1 the value is very far off of the benchmark, the thermionic emission will only work for scenario no. 2 with the sufficient current injection. Therefore, we will first test the steady state current densities for READ in the ON and OFF states for this case.

The results are shown in Fig. 5 in a "Schottky-plot." These data have been created by simulation calculations combining thermionic emission as injection and ejection mechanism with drift-diffusion conduction in the conduction band of the dielectric insulator, ${ }^{11}$ in this case for $15 \mathrm{~nm}$ thick STO with a static dielectric constant $\mathrm{K}(\mathrm{STO})=200$. For the ON state, i.e., the traps are not charged, the current density is in the range of $\geq 10^{4} \mathrm{~A} / \mathrm{cm}^{2}$ for applied voltages $0.2 \mathrm{~V}$ $\leq \mathrm{V}_{\text {appl }} \leq 1 \mathrm{~V}$. For negatively charged traps the current density is decreased (i.e., the resistance is increased: OFF-state) and depends on the polarity of $\mathrm{V}_{\text {appl }}$ because of the asymmetrically distributed trapped charge: If the left electrode (close to the traps) is the cathode the current density is much reduced, by more than a factor of ten in the whole voltage range shown. If the polarity is reversed the condition of a factor of ten reduction in current density for the OFF state is fulfilled for $\mathrm{V}_{\text {appl }} \leq 0.5 \mathrm{~V}$. Hence, for the scenario no. 2 the benchmark conditions for the READ operation for ON and OFF states are satisfied under the assumption that the ON and OFF states are stable during READ (see also Sec. IV).

As shown for scenario no. 1, thermionic emission as injection is insufficient due to the high interface barrier, thus 

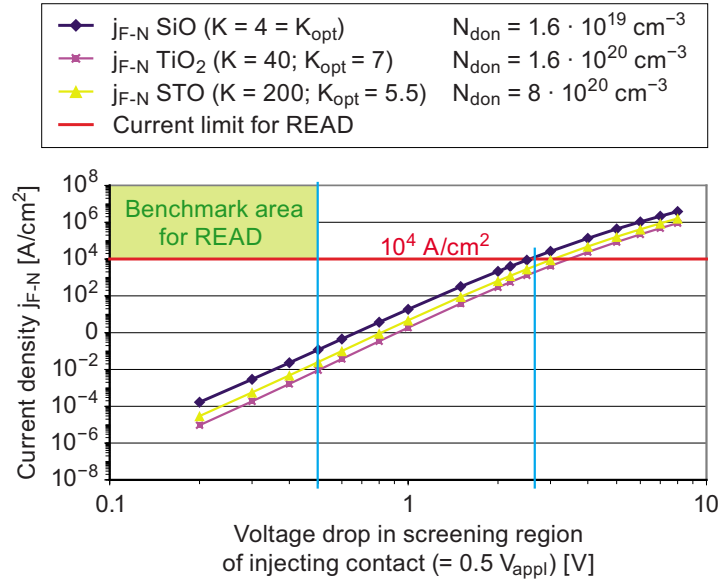

FIG. 6. (Color online) READ operation for scenario no. 1: tunneling injection current density (from cathode) into traps (0.2 eV below CBM) for three different dielectrics with different $\mathrm{K}$ vs voltage drop in the interface screening region.

the current has to be injected by tunneling through the barrier. It can be seen from Fig. 3 that the (parabolic) barrier can be approximated by a triangle; therefore, the FowlerNordheim (F-N) equation for tunneling ${ }^{12}$ has been used for the estimation neglecting differences of effective masses in electrode and dielectric

$$
\mathrm{j}_{\mathrm{F}-\mathrm{N}}=\frac{e^{2} F_{\mathrm{eff}}^{2}}{8 \pi h \Phi_{B}^{\mathrm{eff}}} \exp \left[-\frac{2}{3} \frac{2 \sqrt{2 m_{e 0}}}{\hbar} a_{t} \sqrt{e \Phi_{B}^{\mathrm{eff}}}\right] .
$$

The constant effective electric field $F_{\text {eff }}$ is defined by the potential linearization. The current density was calculated at the Fermi level at the interface, determined by the electrode one, because the tunneling current is a maximum for this energy. The results are shown in Fig. 6 for three different dielectrics with different static and optical K (the Schottky effect for barrier lowering is included making ) and with adjusted donor densities as indicated to achieve the requested barrier width of $6 \mathrm{~nm}$ at zero voltage. The injection tunnelling current density into the trapping states, $\mathrm{I}_{\mathrm{F}-\mathrm{N}}$, plotted over the voltage drop at the injecting interface (which is about equal to half of the applied voltage, $\mathrm{V}_{\text {appl }} / 2$ ), is only sufficient at voltage drops larger than $2.5 \mathrm{~V}$ (corresponding to $\mathrm{V}_{\text {appl }}=5 \mathrm{~V}$ ), but at the maximum benchmark voltage for READ, $\mathrm{V}_{\text {appl }} / 2=0.5 \mathrm{~V}$, the current densities are much too low, at least five orders of magnitude. The corresponding tunneling injection into the conduction band is even lower, about 2 orders of magnitude, due to the increased tunneling width. The dependence on effective mass is cancelled out, because tunneling probabilities for retention and read-out give opposite trends. Therefore, the result can be generalized to all dielectrics. The small dependence in Fig. 6 is due to the different Schottky lowering of the barrier height as a function of the different optical K. Neglecting the "Schottky" barrier lowering would result in a reduction of the current densities of additional orders of magnitude and, thus, aggravate the dilemma.

This exemplifies the voltage-time dilemma for scenario no. 1 already for the READ operation: The current densities can be only enlarged by reducing the barrier width (higher donor densities) or height (electrode selection) to be consistent with $10^{4} \mathrm{~A} / \mathrm{cm}^{2}$ at low voltage read-out, but than the retention times for the trapped charge are drastically reduced, i.e., many orders of magnitude below the benchmark value of 10 years.

\section{THE WRITE/ERASE OPERATIONS}

Only the scenario no. 2 fulfils the benchmarks for readout. Consequently, the WRITE/ERASE operations will be inspected for this scenario only. As stated in Fig. 4 the equilibrium state is the OFF state, i.e., the deep acceptorlike traps are filled with electrons and thus charged. Starting at this OFF state we first test the conditions for WRITE into the ON state, i.e., release the electrons from the traps into available, unoccupied electron states by means of an applied voltage. The benchmark value is $t_{\text {WRITE }} \leq 100$ ns. To exchange the complete trapped charge of $10 \mu \mathrm{C} / \mathrm{cm}^{2}$ within this time a minimum current density, $\mathrm{j}_{\text {WRITE }}$, of $\geq 100 \mathrm{~A} / \mathrm{cm}^{2}$ is required. As the energy difference of the trap energy to available empty electron states in the CB will not be changed by an applied voltage, the only available current path is tunneling through the barrier into available empty states in the close-by left electrode above the electrode Fermi level (using the temperature independent $(T=0)$ approach for the metal band diagram) or into the conduction band if the band diagram is tilted by the applied voltage. To raise the trap level closest to the interface, i.e., $4 \mathrm{~nm}$ apart, to energies $>\mathrm{E}_{\mathrm{F}}^{\mathrm{M}}$ a positive voltage of about $+4 \mathrm{~V}$ has to be applied. The corresponding band diagram is shown in Fig. 7(a). The shape of the tunnel barrier is a trapezoid of width $a_{t}=4 \mathrm{~nm}$ and a reduced effective barrier height much smaller than the nominal $1.55 \mathrm{eV}$ at zero applied voltage [see Eq. (2b)]. The resulting tunneling current densities have been calculated with the F-N tunneling equation [see Eq. (5)] with corrections for direct tunneling through a trapezoidal barrier [see Eq. (2b)] with free electron mass.

$$
\begin{aligned}
& \mathrm{j}_{\mathrm{DT}}=\frac{e^{2}(\Delta V)^{2}}{8 \pi h a_{t}^{2} \Phi_{B}^{\mathrm{eff}}} \exp \left[-\frac{2 \sqrt{2 m_{e 0}} a_{t}}{\hbar} \frac{2}{3} \sqrt{e \Phi_{B}^{\mathrm{eff}}}\right], \\
& \text { with } \Phi_{B}^{\mathrm{eff}}=\left[\frac{\Phi_{B a}^{3 / 2}-\Phi_{B 0}^{3 / 2}}{\Phi_{B a}-\Phi_{B 0}}\right]^{2},
\end{aligned}
$$

$\Delta \mathrm{V}$ is the difference $\left[\mathrm{E}_{\mathrm{T}}(x=4 \mathrm{~nm})-\mathrm{E}_{\mathrm{F}}^{\mathrm{M}}\right] / e$. The results are plotted in Fig. 8 versus $\mathrm{V}_{\text {appl }}$ for the range 4 to $<5.5 \mathrm{~V}\left(j_{\mathrm{T}}\right.$ trapezoidal). The current densities are more than 11 orders of magnitude too small compared to the benchmark value $\left(10^{2} \mathrm{~A} / \mathrm{cm}^{2}\right)$, although they represent the maximum current density by this mechanism under the assumption that the deliverance of trapped electrons to the closest point is not limiting this current further. Therefore, higher voltages have to be applied. For $\mathrm{V}_{\text {appl }} \geq 5.5 \mathrm{~V}$ the barrier shape changes into a triangle, suggesting the use of the F-N formula, Eq. (5), for tunneling with a constant barrier height $(1.55 \mathrm{eV})$, but decreasing barrier width making the states in the conduction band the closest available ones for all the trapped charge with the same conditions [see Fig. 7(b)]. The calculated current densities are also plotted in Fig. 8 ( $j_{\mathrm{T}}$ trapezoidal $)$. Up to $\mathrm{V}_{\text {appl }} \leq 10 \mathrm{~V}$ (i.e., about ten times $\mathrm{V}_{\text {READ }}$ ) the current densi- 
(a)

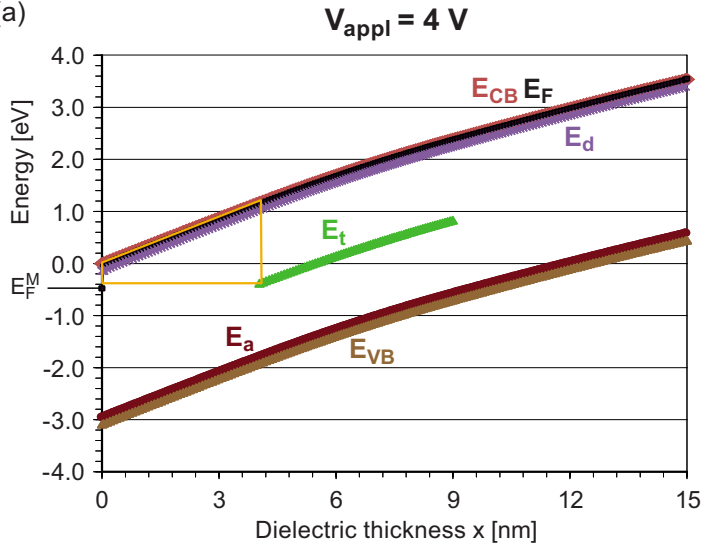

(b)

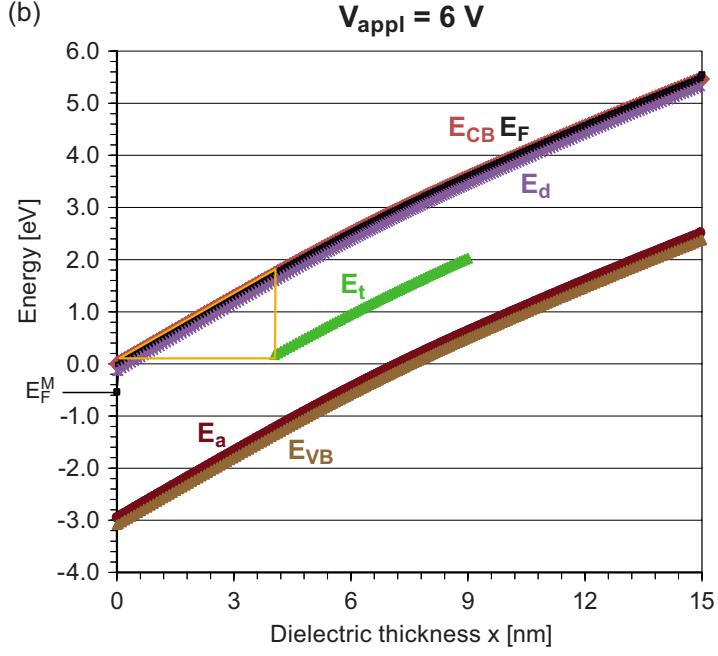

FIG. 7. (Color online) Band diagrams for scenario no. 2 with trapped charge at (a) $\mathrm{V}_{\text {appl }}=4 \mathrm{~V}$ and (b) $\mathrm{V}_{\text {appl }}=6 \mathrm{~V}$, sufficient for tunneling of trapped electrons into unoccupied states above $\mathrm{E}_{\mathrm{f}}^{\mathrm{M}}$ of the (left) electrode or into the $\mathrm{CB}$ of the dielectric. In this range of $\mathrm{V}_{\text {appl }}$ the potential well for the trapped electrons changes from trapezoidal (a) to triangular (b).

ties are (much) smaller than the requested number, but from about $\mathrm{V}_{\text {appl }} \geq 13 \mathrm{~V}$ the current density is sufficient. The released electrons are assumed to be transported away in the CB.

Under the same conditions, a simultaneous "leakage" current is flowing in the conduction band using the simulation calculations as described above. ${ }^{11}$ Its density is at least five orders of magnitude larger than the requested release current density as shown in Fig. 8 ( $j_{\mathrm{CB}}$ leakage). It is worth mentioning that this current density is only a lower limit because of significant tunnel injection into the $\mathrm{CB}$ in addition to the thermionic emission at high applied voltages. Hence, there is a very high density of conduction electrons which may be captured by the unfilled traps which try to reestablish their equilibrium state, the charged one! Only if the probability for trapping of the conduction electrons is much smaller than $10^{-6}$ compared to the release probability (this would be unreasonable for "trapping" defects!) the traps would be discharged to a considerable amount. In turn, if this would be the case this would give problems with the ERASE operation, i.e., re-charging the traps, because the erase current density has to be enhanced by the same factor $\left(10^{6}\right)$ due

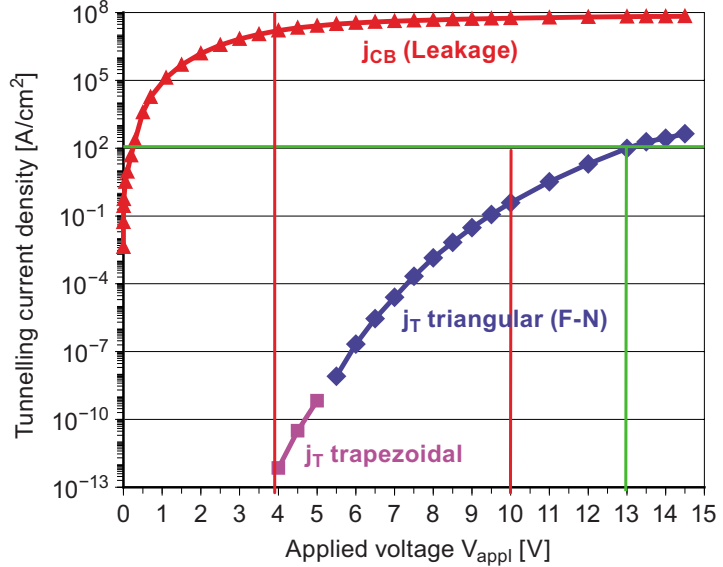

FIG. 8. (Color online) WRITE operation for scenario no. 2: tunneling current densities for WRITE, (i.e., the release of the trapped charge in $E_{t}$ ) vs applied voltage, $\mathrm{V}_{\text {appl }} \cdot \mathrm{V}_{\text {appl }}<4 \mathrm{~V}$ : no significant tunneling due to $\mathrm{E}_{\mathrm{t}}(x$ $=4 \mathrm{~nm})<\mathrm{E}_{\mathrm{f}}^{\mathrm{M}} ; 4 \mathrm{~V} \leq \mathrm{V}_{\text {appl }}<5.5 \mathrm{~V}$ : tunneling through trapezoidal barrier; $5.5 \mathrm{~V} \leq \mathrm{V}_{\text {appl }}$ : tunneling through triangular barrier. For comparison, the leakage current density in the conduction band is also shown.

to the bad trapping efficiency and this will then drive the current density for ERASE far off all realistic numbers.

For scenario no. 2 the voltage-time dilemma appears in the WRITE/ERASE operation. This result can be generalized also for other switching insulators under the same condition, i.e., neutral contacts with low interface barrier with deep in-gap traps.

\section{SUMMARY AND CONCLUSIONS}

The trapping-detrapping of electronic charge at immobile traps in insulators as a mechanism for a nonvolatile resistive switching in MIM thin films stacks was investigated under benchmark values competitive to present-day FLASH memories. It was shown that under these conditions this "pure electronic" model-the well-known Simmons \& Verderber model is very similar to a one of the possible variants (scenario no. 1) - faces an insolvable voltage-time dilemma. The large retention time of 10 years for nonvolatile memories calls for sufficient barriers to suppress the escape of the trapped electronic charge by thermally activated or tunneling processes. At the postulated low voltages these necessary barriers prevent high enough current densities needed for short READ and WRITE pulses ( $\leq 100 \mathrm{~ns})$ in the small areas $\left(\leq 10^{-10} \mathrm{~cm}^{-2}\right)$ of ultrahigh integrated memories. At the postulated current densities (at much larger applied voltages) the nonequilibrium charge state of the traps is severely affected and thus unstable on time scales which are many orders of magnitude smaller than the required retention time. This dilemma has been discussed in details for two different scenarios with different insulator band and defect properties, but it is general to all possible MIM thin film stacks because of basic physical reasons under the given (benchmark) conditions.

${ }^{1}$ J. G. Simmons and R. R. Verderber, Proc. Roy. Soc. A301, 77 (1967).

${ }^{2}$ H. J. Hovel and J. J. Urgell, J. Appl. Phys. 42, 5076 (1971).

${ }^{3}$ A. Odagawa, H. Sato, I. H. Inoue, H. Atoh, M. Kawasaki, and Y. Tokura, Phys. Rev. B 70, 224403 (2004). 
${ }^{4}$ C. J. Kim and I. W. Chen, Thin Solid Films 515, 2726 (2006).

${ }^{5}$ C. Pearson, J. H. Ahn, M. F. Mabrook, D. A. Zeze, M. C. Petty, K. T. Kamtekar, C. Wang, M. R. Bryce, P. Dimitrakis, and D. Tsoukalas, Appl. Phys. Lett. 91, 123506 (2007).

${ }^{6}$ K. K. Likharev, Appl. Phys. Lett. 73, 2137 (1998); Nanotechnology 10, 159 (1999).

${ }^{7}$ L. D. Landau and E. M. Lifshitz, Quantum Mechanics (Addison-Wesley, Massachusetts, 1958), p. 171.
${ }^{8}$ K. C. Kao and W. Hwang, Electrical Transport in Solids (Pergamon Press, Oxford, UK, 1981), p. 148.

${ }^{9}$ W. Hofman, S. Hoffmann, and R. Waser, Thin Solid Films 305, 66 (1997). ${ }^{10}$ R. Waser, NATO ASI Ser., Ser. E Appl. Sci., Vol. 284, 223 (1995).

${ }^{11}$ H. Schroeder, S. Schmitz, and P. Meuffels, Appl. Phys. Lett. 82, 781 (2003).

${ }^{12}$ R. H. Fowler and L. Nordheim, Proc. R. Soc. London, Ser. A 119, 173 (1928). 